\title{
Agroecologia: Una plataforma para la Paz
}

\section{Agroecology: A platform for Peace}

\author{
Wilson Miguel Salas Picón
}

Universidad Cooperativa de Colombia

wilsonsalas2@gmail.com

\section{Elsa Beatriz Valenzuela Bonilla}

Universidad Cooperativa de Colombia

elsa.valenzuela@campusucc.edu.co

\section{Alberto Prada Galvis iD}

Universidad Cooperativa de Colombia

alberto.pradag@campusucc.edu.co

\section{Resumen}

Este artículo presenta resultados a nivel documental sobre los aportes de la agroecología para la construcción de escenarios para la Paz en Colombia. Para ello se toma como referencia la experiencia de algunos países, y se presenta el camino recorrido. A nivel exegético se manifiesta la agroecología como un modelo de intervención tripartito (cienciaambiente, práctica, intervención sociopolítica), fundamentado desde una dimensión socioecológica y desarrollo sostenible. Se concluye que, para el caso de Colombia, este modelo representa una propuesta para la Paz dada su aplicabilidad desde el enfoque territorial. Finalmente, se plantea una descripción de la realidad sociopolítica-rural, y se identifica la necesidad de articular diversos actores para su implementación al igual que políticas públicas que mejoren las condiciones del agro colombiano.

Palabras clave: Agroecología, Posconflicto, Paz, desarrollo territorial, Agricultura.

\begin{abstract}
The article presents results at the documentary level on the contributions of agroecology for the construction of scenarios for Peace in Colombia. For this, the experience of some countries is taken as a reference and the path taken is presented. On an exegetical level, agroecology is manifested as a model of tripartite intervention (science-environment, practice, sociopolitical intervention), based on a socio-ecological dimension and sustainable development. It is concluded that, in the case of Colombia, this model represents a proposal for Peace given its applicability from the territorial approach. Finally, a description of the socio-political-rural reality is presented and the need to articulate various actors for its implementation is identified, as well as public policies that improve the conditions of Colombian agriculture.
\end{abstract}

Keywords: Agroecology, Post-Conflict, Peace, Territorial Development, Agriculture.

Articulo: Recibido el 7 de junio de 2019 y aprobado el 22 de octubre de 2019

Cómo citar este artículo:

Salas Picón, W.M., Valenzuela Bonilla, E.B. \& Prada Galvis, A. (2019). Agroecología: una plataforma para la Paz. Reflexión Politica 21(43), pp. 79-91. doi. 10.29375/01240781.3634 


\section{Introducción}

La tierra ha sido el espacio para la convergencia y el desarrollo de procesos que han conllevado al planteamiento y puesta en marcha de estrategias socioeconómicas para la subsistencia del ser humano y, con ello, el origen de sociedades sustentadas en la actividad agrícola.

El ejercicio de la agricultura en palabras del Grupo temático "Soberanía alimentaria y agricultura familiar" de la institución Coordinadora de Entidades Extranjeras de Cooperación Internacional (en adelante, COEECI) (2014), permitió la conformación de las primeras sociedades, así como el establecimiento de valores culturales (fiestas, canciones, costumbres) que facilitaban su interacción y la construcción de su identidad.

Indudablemente, la agricultura ha posibilitado la construcción de tejido social a partir de las dinámicas del trabajo asociativo que convergen para alcanzar múltiples objetivos, destacándose: producción de alimentos como estrategia de seguridad alimentaria, distribución de tierras, modelos económicos y desarrollo social, entre otros. No obstante, el incremento de la tasa de natalidad de la población humana, los modelos económicos de desarrollo implementados por los países a nivel mundial, la tenencia de la tierra (propiedad) y el desarrollo rural se han convertido en factores de riesgo y depredación que vulneran el equilibrio de la relación Hombre-Tierra-Sociedad y con ello, el origen de múltiples conflictos. Frente a lo expuesto, se han generado estrategias, materializadas en acciones desde diversas disciplinas, las cuales buscan disminuir el impacto que ha tenido la actividad humana sobre la explotación de la tierra y su tenencia, el manejo de los recursos naturales y la implementación de sistemas de desarrollo que tienen como objetivo la preservación del medio ambiente; la seguridad alimentaria, la asociatividad, las relaciones de poder, entre otros pilares que pretenden garantizar la vida (INCAP, 1998).

La agroecología, la agricultura familiar y la agricultura ecológica son sistemas de gestión y desarrollo que han surgido como alternativas para hacer frente a los factores de riesgo generados por modelos económicos y políticas depredadoras que poseen elementos comunes a nivel etiológico en el surgimiento de la violencia sociopolítica en Perú y Centroamérica. Estas propuestas comparten un mismo denominador -promoción de prácticas agrícolas sostenibles desde una dimensión homeostática entre producción y cuidado de la naturaleza, justicia social, la identidad y cultura, visibilización de lo rural y defensa de la tierra y el territorio-, pero sus bases epistémicas, establecen líneas diferenciales. Ahora bien, las iniciativas expuestas no han surgido por un interés político, ni por políticas gubernamentales o por el resultado de un trabajo bajo la metodología de corresponsabilidad por parte del gobierno en Perú y Centroamérica, sino por la apuesta social y de desarrollo que han realizado ONGS o asociaciones, $u$ otras organizaciones, apoyadas con recursos de cooperación internacional, las cuales pretenden brindar alternativas para la implementación de modelos y estrategias que no sólo preserven el medio ambiente, sino también que fortalezcan la economía, el tejido social y la tenencia de la tierra. El trabajo realizado por las diversas organizaciones permitió la visibilización de su relevancia y, con ello, la movilización de actores gubernamentales e intereses políticos que dieron origen a ministerios, legislación y otros entes, como es el caso de Perú.

Ahora bien, no existe un consenso que permita rotular en un mismo concepto la agroecología con la agricultura ecológica, agricultura de base agroecológica y agricultura familiar, dado que han proliferado un sinnúmero de definiciones al respecto; no obstante, se cuenta con pilares y principios que permiten definir con claridad este campo: es un estilo cultural y de vida, tiene interrelación con factores sociales, comunitarios, culturales, políticos, económicos; la familia y las comunidades son fundamentales; la minimización de riesgos en la producción -preservación, inclusión y reconocimiento del rol de la mujer (enfoque diferencial y de género), es diversa en sus actores, en lo referente a las actividades y acciones; parte del conocimiento ancestral (diálogo de saberes), relación directa entre productor- consumidor (seguridad y soberanía alimentaria); es una agricultura alternativa, representa un movimiento sociopolítico y, finalmente, es un nuevo escenario epistemológico, de innovación social y tecnológica que plantea un escenario glocal -prácticas y epistemologías que se construyen desde los diversos territorios(COEEC, 2014). 
En lo referente al tema de movimiento sociopolítico, se evidencia la conformación de organizaciones de base que plantean cambios significativos en el abordaje de problemáticas desde el enfoque de la agricultura sostenible y de preservación. Algunas de ellas son: Movimiento Social Campesino a Campesino en Centroamérica, Cosecha en Honduras, EDAC en Perú, comités de desarrollo, entre otros, los cuales se conformaron con el objetivo de contrarrestar los fenómenos de desigualdad y pobreza, o de protegerse de la violencia, como es el caso del EDAC y Sendero Luminoso, de igual forma que el caso de las mujeres Salvadoreñas que se organizan desde la perspectiva del enfoque territorial y la agricultura en el periodo de transición de la post-guerra (Navas, Orellana \& Domínguez, 2000). Indudablemente, los grandes pasos que se han dado en este escenario han facilitado la consolidación de procesos, modelos pedagógicos y de aprendizaje (centrados en los saberes ancestrales o en la gente), estructuras organizativas y de gestión, alteridad, es decir, el reconocimiento del Otro (incluida la tierra).

Por otro lado, Alvarado De La Fuente (2000), en su artículo "Siete retos de la agroecología: desde la experiencia en el Movimiento agroecológico peruano", expresa que han dado grandes avances en los siguientes pilares: "La transición, Metodologías participativas, La participación de la mujer, Productividad y rentabilidad, Indicadores de impacto, Mercado y gestión, Movimiento agroecológico" (p. 231). El último representa el más fuerte, ya que han proliferado un sinnúmero de organizaciones, pero todas trabajan de manera individual y desarticulada. Sin embargo, argumenta que el trabajo de once años ha permitido la consolidación de procesos de igual manera que la conformación de la Red de Agricultura Ecológica (RAE). Es importante señalar que Perú es uno de los países de Suramérica que ha realizado un trabajo arduo en lo referente a la distribución de la tierra y el fomento de la agroecología (Alvarado De La Fuente, 2000; Altieri \& Toledo, 2010).

El desarrollo de un modelo rural donde la relación Hombre-Naturaleza es fundamental, ha dado grandes pasos y transformaciones en países de Centroamérica y en Perú, permitiendo generar un reconocimiento e importancia del mismo modo que el planteamiento de estrategias que abordan problemáticas que conllevan al origen de la violencia sociopolítica. Es de resaltar que es poca la literatura en donde se plantea la aplicación de este modelo de desarrollo como propuesta en un escenario de post-acuerdo o finalización del conflicto armado. A continuación, se realizará un recorrido sobre la propuesta, conceptos y modelos en países como Perú y algunos de Centroamérica, a manera de referente sustancial para asimilar su dinámica en el contexto de las experiencias analizadas en las tres regiones objeto del estudio, con sus particularidades, en el Magdalena Medio colombiano, no sin antes hacer una reflexión breve sobre las implicaciones del modelo.

\section{Algunas consideraciones sobre Agro: Familiar y Ecológica}

El planteamiento de nuevas dinámicas de desarrollo en las zonas rurales hacia la búsqueda de una paz estable, duradera y sostenible, implica que se piense en métodos de recuperación del campo que se conviertan en vías amplias y alternativas en el posconflicto. Para ese fin, se deben repensar los conceptos que históricamente han estado vinculados con la práctica de la agricultura y el rol de los campesinos como sujetos políticos. Se hace necesario que el desarrollo rural propuesto en los acuerdos de paz firmados en La Habana, y en la reforma agraria, incorpore en los contextos particulares nuevas formas para trabajar el agro (homeostasis del sistema y subsistemas que lo sostengan), en consideración de las dimensiones territoriales, sociales, económicas, ecológicas, solidarias y tecnológicas, hasta alcanzar una renovación sistémica y sostenible (Sarandon, 2011). Esa perspectiva supone un reconocimiento agrícola desde un punto de vista inter y transdisciplinario a nivel productivo, y una visión holística de todo lo que involucra lo agrario; el reconocimiento del umbral de las necesidades y el impacto en los campesinos que fueron despojados de sus territorios, y que ahora retornan a sus tierras, conlleva a repensar la praxis de la agricultura en el país en las siguientes dimensiones: desarrollo con enfoque territorial, integridad, conservación de los recursos, sustentabilidad financiera y ecológica (Sarandon \& Sarandon, 1993).

En coherencia con lo expuesto, los modelos alternativos que se planteen han de separarse de las prácticas de la agricultura convencional que fue impuesta por la Revolución Verde, que tuvo en manos del monopolio armado y de los latifundistas 
del capitalismo una fuerza detractora en contra del campesino que fue desplazado. Estos modelos alternativos buscarían reducir los impactos sobre los ecosistemas, a favor de una agricultura más humana, solidaria y sostenible. La premisa planteada amerita un trabajo mancomunado entres organizaciones de familias campesinas, Estado (gobiernos territoriales), el sector empresarial y la academia. Desde este horizonte, se entiende que los nuevos modelos agrícolas que se vinculen a las acciones del posconflicto deben ver los procesos como un todo muy complejo que incluye temas como: semillas, el riego, la producción de alimentos, las materias primas, los factores ambientales, la tecnología aplicada y todo aquello que redunde en beneficio de los ecosistemas, de tal manera que se fortalezca el equilibrio entre estos y la cultura humana desde una perspectiva solidaria.

La ciencia agroecológica se ubica precisamente en los planteamientos que se han venido esbozando en relación con el equilibrio que se espera en los agrosistemas, relación HombreNaturaleza, y surge como una de las alternativas a la agricultura convencional. Se trata de todo un movimiento que se basa en principios ecológicos, éticos y de cultura ambiental. Pretende el manejo de sistemas agroalimentarios sostenibles que establezcan un diálogo entre los conocimientos tradicionales y los avances que sobre el desarrollo agrario integral se han venido generando. Se trata de llevar a la praxis y hacer converger los principios y conceptos para la implementación y gestión de agrosistemas sostenibles, lo cual permite la ampliación de su biodiversidad funcional, es decir, que los sistemas de cultivo reduzcan el uso de insumos externos y se provean de las ventajas biológicas que la misma naturaleza brinda. No de otra manera podría pensarse en una agricultura que incentive la soberanía alimentaria y proporcione el tiempo suficiente para que los agrosistemas puedan alcanzar altos niveles de resiliencia.

Por lo anterior, se plantea un modelo con un enfoque innovador, denominado agroecología y se postula como una fuente de conocimiento. $\mathrm{Su}$ base se sustenta a partir del trabajo realizado por las comunidades rurales, las cuales le aportan los principios tradicionales de la agricultura, en tanto que, al mismo tiempo, se van incorporando los avances contemporáneos en ciencia y tecnología agronómica, generando sinergias en la producción. De otra parte, este modelo, además de manejar la biodiversidad presente en los agrosistemas locales y ecozonas, potencia los recursos sociales y culturales; permite un empoderamiento de la producción en grande por parte de los campesinos quienes pondrían en práctica sus conocimientos para hacer frente al cambio climático a través de la difusión de estrategias que históricamente han aplicado y quizás se reduzca en gran parte la pobreza rural y el uso de los agentes agroquímicos. Otra ventaja de este sistema es que diversifica los medios de vida mientras fortalece el tejido social; enriquece saberes y praxis, tanto ancestrales como tradicionales, en lo referente a la relación Hombre-Naturaleza, utilización del patrimonio natural, redefiniendo las relaciones campo-ciudad mediante el acceso más fácil a los mercados. Desde esa perspectiva, es connatural al proceso pensar en una cadena de producción autónoma, cuya soberanía alimentaria defienda la ruptura con las acciones de intermediación, y sea el mismo campesino quien lleve sus productos directamente a los centros urbanos. Así pues, el camino de la producción se verá fortalecido por unas estrategias de transformación y distribución, tan efectivas y confiables que generen una mayor estabilidad a los campesinos y al sistema agroecológico.

La agroecología es un campo del saber que plantea un escenario disciplinar científico que articula conocimiento, no sólo de la agronomía y ecología, sino también desde otras disciplinas como la antropología y la sociología, lo que permite tener una mirada integral y sistemática para la generación, validación y aplicación de saberes desde una perspectiva ética y amigable desde agroecosistemas sostenibles (Sarandon, 2002). Para GuzmánCasado, González de Molina, y Sevilla-Guzmán (2000), las prácticas responden a principios como el enfoque holístico y sistémico, lo que significa que los campesinos asumen el compromiso de ser sujetos que auscultan las problemáticas de sus tierras y presentan alternativas de solución (acciónparticipación). Así pues, la sostenibilidad se plantea como la posibilidad de explorar constantemente puntos homeostáticos, es decir, de equilibrio entre las dimensiones que componen el sector agrícola y la preservación de los recursos, ya sea ésta de carácter social, cultural, económica, política, ética y ecológica (Caporal \& Costabeber, 2004).

En este sentido, la agroecología puede interpretarse como un movimiento que desafía los saberes científicos convencionales y los postulados 
de la agricultura industrial establecida por la revolución verde. En esa medida, el propósito recoge el clamor y las prácticas tradicionales de las comunidades campesinas quienes han luchado para contrarrestar los flagelos sociales (exclusión, pobreza, desigualdad), económicos, culturales, ambientales (devastación del ambiente), entre otros; se desprenden de un modelo geopolítico neoliberal y post-industrial de desarrollo que promueve políticas afectando el área rural. Eso significa que su acción va encaminada a vivificar en el campo una gestión por la conservación del agua y semillas criollas, el manejo adecuado de la tierra; igualmente, el cuidado y salubridad de los cultivos, la organización de redes a nivel de comercialización, cuyos actores principales sean los productores, el acercamiento efectivo con los consumidores y la oposición a los organismos genéticamente modificados, a las transnacionales de los agroquímicos y al agrocombustible incursionando en ecología política y proclamando la solidaridad entre los pueblos (Mejía, 2014).

En ese mismo marco, surge una economía campesina y comunitaria, que busca posicionarse y lograr el reconocimiento a nivel agrario como pilar para el desarrollo integral en el posconflicto. Eso es, la promoción de un sistema agroecológico que se sustenta de manera ecléctica desde los aportes de diversos modelos de agricultura, el cual reconoce la diversidad, fomenta sistemas agroalimentarios, la inclusión y la sostenibilidad. Consecuentemente, alrededor de este modelo se caracterizan aspectos muy particulares como el arraigo por la tierra, la seguridad alimentaria, la producción y consumo de comida tradicional cultivada con ingredientes directamente cosechados de las huertas familiares; de igual forma, la preservación y uso de los recursos naturales, los niveles de empleabilidad y la bio-diversidad agrícola. Este enfoque pretende armonizar el funcionamiento de los sistemas naturales en relación con lo que es mejor para el habitante del campo y para los consumidores en general, e incluye el enfoque de género (las mujeres juegan un papel fundamental en la siembra de semillas como guardianas de la diversidad y los recursos genéticos). Su política es proveer a las familias y a los consumidores alimentos saludables, con rendimientos sostenibles, sin detrimento de los recursos naturales y es un ingrediente para contrarrestar el umbral de la pobreza y el hambre. Se considera desde esta perspectiva como un elemento importante para el progreso desde un enfoque territorial, dado que dinamiza procesos y beneficia a la familia rural, lo cual se traduce en seguridad alimentaria, nutrición efectiva y la conservación de la agro biodiversidad (FAO, 2007). Es un propósito esencial de esta propuesta que las empresas no controlen la cadena alimentaria, sino que esta tarea le corresponde exclusivamente a productores y consumidores.

En la actualidad, los diversos procesos de la agricultura han sido mejorados a través de las nuevas tecnologías. El trabajo articulado entre el saber campesino y el saber científico (tecnologías amigables al ambiente), permiten elevar los niveles de sustentabilidad de la tierra desde un enfoque de conservación del medio ambiente. La protección del medio ambiente y la reutilización de los materiales en el ciclo biológico se convierte en una bandera para la preservación de la especie (Boza-Martínez, 2010). Todo esto conduce, necesariamente, a desarrollar hábitats rurales teniendo en cuenta los recursos del territorio. Ese potencial endógeno ha de ser identificado en cada porción de la naturaleza y de la sociedad; no obstante, dicho potencial no puede negarse ni reemplazarse por el sistema industrial (Sevilla- Guzmán, 2006). Ahora bien, algunos teóricos establecen un marco de diferenciación entre modelos de agricultura y agricultura ecológica. Las propuestas permiten evidenciar diferencias a nivel sociológico al igual que la episteme de este y su influencia en la gestión del medio ambiente (BozaMartínez, 2010).

En 1995 el Panel Nacional de Estándares Orgánicos (NOSB), dependiente del Departamento de Agricultura de los Estados Unidos (USDA), precisó que la agricultura ecológica es un sistema de gestión ecológica que promociona la preservación del medio ambiente y el mejoramiento de la biodiversidad, la tierra y biología de las zonas. Su base se sustenta sobre el empleo de materiales e insumos existentes en el territorio, el equilibrio en la relación interdependencia Hombre-Naturaleza, el no empleo de químicos y la optimización de la productividad, del mismo modo que la salud en las poblaciones campesinas (Boza-Martínez, 2010). Por el contrario, el concepto de agricultura familiar tiene su base en las comunidades que han estado deprimidas históricamente en los países latinoamericanos, reducido a las huertas, las pequeñas granjas y la economía campesina. 


\subsection{Experiencias en Perú y Centroamérica}

Perú y países centroamericanos han padecido, de igual forma que Colombia, los efectos de la violencia sociopolítica. Como resultado, en función del posconflicto y su experiencia en el agro, decidieron establecer dentro de sus prioridades unas agendas estratégicas cuyo núcleo fundamental se centra en conceptos de Seguridad Alimentaria y Nutricional (Cortez-Dávila, 2016), al igual que soberanía alimentaria. Estos se fundamentan no sólo en la capacidad alimentaria (producción y disponibilidad) de las regiones afectadas por la situación de confrontación, reafirmando la visión de los Derechos Humanos, sino también en la definición de políticas agrarias con base a las capacidades locales. Sus experiencias dejaron una enseñanza en la estructura productiva, la comercialización, los factores productivos, tales como el suelo, el crédito, el agua, la tecnología y los recursos humanos. Igualmente, en lo relacionado con la situación ecosistémica, biodiversidad, clima y genética; condiciones políticas y sociales, comercio y producción. En principio, para que este ideal se estableciera, se tuvo y se ha tenido que luchar contra una inseguridad alimentaria creciente en esos territorios, una pobreza cabalgante en el sector rural y unos índices de desnutrición creciente en el mismo ámbito (CortezDávila, 2016). Tal situación requirió proyectos de escalonamiento de experiencias agroecológicas que consideraran las diferentes dimensiones de estos procesos de recuperación de la actividad agrícola y del campo, el cual había quedado maltrecho por motivo de la guerra. Con Sarandon y Flores (2014), se explican dichas dimensiones (en esa dinámica hubo intervención operativa de diversas entidades, públicas y privadas, con prioridad para las organizaciones sociales de los sectores rurales, entre los cuales se muestran los promotores, los agricultores, los líderes innovadores y las familias). A continuación, se presentan sus características:

A nivel social se buscó, y aún persiste, elevar el índice de equidad en la distribución de recursos, con injerencia intergeneracional, entre los beneficiarios de las generaciones actuales y futuras. De esa manera, la población, que trabaja la tierra y produce alimentos para el resto del mundo, asegura una mejor calidad de vida y se avanza hacia la reconstrucción de lo rural desde el trabajo colectivo, lo cual robustece y establece un camino hacia el desarrollo, fomento y mantenimiento del capital social.

En el componente cultural se estima que el modelo considera importante establecer como punto de partida para la fundamentación de las propuestas y políticas de desarrollo, el saber ancestral y local de las comunidades rurales. Esta dimensión es tenida en cuenta para la producción de conocimiento y de tradición agrícola.

En cuanto a la dimensión económica, se consideran las características del mercado, los costos de los insumos, los riesgos asociados con la diversificación de productos y la comercialización.

En lo referente a la política, se entienden como relevantes para el mejoramiento del área ruraly producción agrícola, las dinámicas de participación y democracia, al igual que la representación de los segmentos de la población en lo concerniente a las elecciones y decisiones sobre cuestiones de la subsistencia agrícola. Finalmente, el proceso requiere vínculos morales, en su dimensión ética, como es la conservación, preservación de la naturaleza y su respeto; igualmente, la preservación de valores, la reivindicación de la ciudadanía y el restablecimiento de la dignidad de los campesinos.

Las dimensiones expuestas constituyen un camino hacia la recuperación de la vida agrícola.

Un hecho importante que constituye un común denominador de las experiencias en Perú y en los países centroamericanos, el cual se convirtió en un modelo generalizador, fue la práctica y difusión de la capacitación campesino a campesino. Esto como resultado de los procesos de formación que se emprendieron a nivel académico en las universidades, que luego se transfirió a comunidades más vulnerables. En ese sentido, las entidades académicas de investigación jugaron un papel especial. Hubo formación de los recursos humanos en gran parte del territorio; se analizaron en grupos de discusión las propuestas dirigidas hacia el cambio y, lo más valioso y rescatable, una inserción agresiva en las comunidades de agricultores con una doble intención: aprender de la agricultura tradicional en las localidades, divulgándolas en múltiples espacios y, segundo, llevar nuevas tendencias en materia de conocimientos de agronomía y nuevas tecnologías. Fue esencial, además, la contribución que se le dio a los procesos mediante la validación de la agroecología. Otra característica para reconocer fue la vinculación de los agricultores 
de manera directa en el mercado, fortaleciendo un escalonamiento agroecológico que basaba su accionar desde dos dimensiones: el sendero de la organización cooperativa y la apertura directa en el mostrador de venta (mercados agroecológicos y campesinos). De hecho, esta estrategia mejoró los beneficios económicos y de seguridad en estos países, mediante una comercialización de los productos agropecuarios de una forma más amplia, con precios diferenciados según la dinámica de oferta desarrollada.

El cambio no surgió sin preparación; fue necesaria toda una maduración que se dio a propósito de los saberes de los agricultores tradicionales, la reflexión desde la academia, y el diagnóstico de las necesidades en los territorios. En esa perspectiva, se caminó por: a) un período de transición en el cual los agricultores rompen con el sistema convencional de la revolución verde y se inician en el sistema agroecológico; b) unas metodologías participativas que buscan la integración de comunidades de campesinos alrededor de objetivos concertados, de planes de capacitaciones en principios y técnicas agroecológicas, como sucedió en el Perú con la Red de Agricultura Ecológica entre 1991 y 1996; c) la participación de la mujer entendiendo que nada es sin la presencia de esta como sujeto de acciones, dinamizando el elemento cultural de la equidad de género; d) la productividad y la rentabilidad, planteando nuevos escenarios emergentes desde el sector cooperativo para que las ganancias se convirtieran en una posibilidad atractiva; e) los indicadores de impacto que sirvieron como referente para evaluar los resultados positivos de la agroecología.

En Perú, la tarea no fue ni ha sido fácil en el afán de orientar el movimiento agroecológico, entre otras, por sus implicaciones políticas, puesto que la labor de unir al Estado, la empresa privada, a las organizaciones y a las comunidades de campesinos en todo el territorio ha sido compleja. Por ejemplo, un gran paso fue el diálogo y la articulación generada entre comunidades y organizaciones campesinas que se conformaron como una estrategia de resistencia al conflicto armado, a partir de proyectos y de la conformación de microempresas como unidad básica de asociación (Ranaboldo y Venegas, 2007). Entre las bondades de este vínculo se reconocen factores como el aumento de la productividad, el concepto de la propiedad en lo referente a la tenencia de la tierra, dado que muchos suelos fueron otorgados en usufructo por el Estado para que agricultores pudieran administrar, gestionar, diversificar el agrosistema y conservar la tradición campesina. Se capacitó el recurso humano de manera transversal con acciones como ferias agropecuarias, talleres y cursos sobre agroecología, sin olvidar el trabajo de las universidades con la inclusión en sus pensum académicos de temas agroecológicos, lo cual produjo la creación de microempresas conformadas por campesinos asociados. En consecuencia, fue clave mantener ofertas formativas continuas $y$ calificadas, desarrollar innovación permanente y medir la efectividad de los procesos agrícolas centrados en la resiliencia de la tierra y el desarrollo agrícola centrado en la gente. La realidad expuesta, evidencia que los sistemas productivos que se implementen se caracterizaran por: conservación de los recursos naturales, diversificación del cultivo y vegetación, sustentabilidad-producción, empleo de saberes sociales y científicos, formación de redes para la gestión y acompañamiento (Alvarado, 2012; Altiere, 1989).

Ahora bien, el modelo de agricultura en el Perú ha tenido una importante evolución en aspectos tales como: promoción y formación, asociatividad, empleabilidad, generación de redes, aporte científico, sinergias institucionales, conservación, sistemas de cultivo y compost, tenencia de la tierra, rescate del saber campesino, el planteamiento de un plan de acción desde la Comisión Coordinadora Nacional sobre Agricultura Ecológica (Wú-Guin y Alvarado de la Fuente, 2008). Para el año 2000 se presentó un hito dentro del proceso; se conformó la Asociación Nacional de Productores Ecológicos, con asociaciones regionales, en provincias y distritos. Además, se impulsaron estrategias de mercadeo y comercialización de los productos certificados como ecológicos a nivel local. Durante este período, los campesinos han pasado a ser actores valorados dentro de esta dinámica y se ganan un espacio sustancial en los mercados para el posicionamiento de los productos. Se inicia el Proyecto in Situ mediante el cual se pretende conservar, preservar y recuperar recursos biogenéticos. En este punto es necesario mencionar el estudio cualitativo realizado por el Instituto Oficial para la Investigación de la Calidad de Alemania, desde una perspectiva longitudinal (12 años), el Instituto evidenció que existen mayores bondades a nivel nutricional en los productos donde se emplearon los procesos 
de la agricultura ecológica (RAE, 1990; citado por Alvarado De la Fuente 2004).

Hoy en día, la agricultura peruana se sigue alimentando de los aportes de la academia y del saber producido por los campesinos y transmitidos a través de la metodología campesinocampesino, mediante un diálogo productivo en un país multicultural que se enfrenta a las políticas neoliberales. Para Felipe-Morales (2001), más allá de ese inconveniente, la agricultura que promueve la autonomía, el saber del campesino, la defensa de la tierra, el equilibrio entre producción y preservación es la mejor alternativa, sobre todo para los pequeños $\mathrm{y}$ medianos agricultores que han sido marginados. Ellos tienen la posibilidad de no depender de insumos externos por lo que disminuye el costo de la producción y mano de obra. Finalmente, se resaltan las experiencias y camino recorrido en países de Centroamérica y Latinoamérica (Romero, 2014), tales como: Cajamarca (Alvarado De la Fuente y Wiener, 1998); Alto Piura (Alvarado, 2001); Penipampa (Alvarado, 1993); Finca Monte Fértil (Alvarado de la Fuente, 2004); Alta Verapaz Galvéz et al., (2015); Guatemala (Dürr, 2015); México (Toledo, 2012); Nicaragua (Amaya-Palacios, 2013); Costa Rica (Baumeister, 2010); Carrazón, (Corleto y Sibrián, 2013; Fernández, 2011).

\section{Trascender de la agricultura a la agroecologia: Insumos para la paz}

En la historia de la humanidad, la agricultura nace por la necesidad de sobrevivir y organizarse para obtener sostenibilidad a largo plazo en la generación de combustibles fósiles, la caza de animales y la recolección de plantas; pensando, posteriormente, en satisfacer sus necesidades básicas con un proceso de manejo y selección, tanto de las plantas que recolectaba, como de la ubicación de terrenos fértiles. Así mismo, el descubrimiento y uso del fuego posibilitó quemar biomasa para atraer a los animales de caza, sin percatarse que la quema indiscriminada de vegetación posteriormente afectaría el ecosistema; este evento se conoció como el primer sistema de producción de cosechas, en el cual participaban los diferentes integrantes de las comunidades nómadas, quienes asumían tareas y roles específicos en los periodos de siembra, cultivo y recolección de productos (Pérez, 2001). En este proceso primario, la germinación de las semillas se desarrolló de manera fortuita y aleatoria en silos, práctica que, en el transcurso del tiempo, muestra el camino para la siembra. Del mismo modo, la técnica de observación y el conocimiento empírico sobre los cambios climáticos permitió la creación de un calendario que identificaba las épocas donde los cultivos podrían ser productivos y los tiempos propicios para el almacenamiento de agua dulce y conservación dealimentos suficientes, para subsistir en periodos secos. La siega y aprovechamiento de los sembrados implicó la utilización de barretas y mazos de madera que facilitaban la extracción de raíces en las especies perennes, es decir, se originan avances en la tecnificación y utilización de herramientas (Denardi, 2001).

Las reflexiones teóricas sobre las relaciones biunívocas de los seres humanos y el medioambiente, los sistemas de producción intensiva y extractivista, su impacto bio-psicosocial y ecológico, conllevaron a plantear un modelo de agricultura que abordara lo social, la relación Hombre-Naturaleza, la sostenibilidad ambiental y sustentabilidad productiva, conciencia política, elementos que transcienden el territorio desde lo agrícola (Ruiz-Palacios, 2017). Lo expuesto, brindó las bases para la ampliación de los límites de la agricultura moderna, basándose principalmente en investigaciones científicas sobre cómo plantas y animales responden e interactúan con el medio ambiente desde una comprensión holística entre el medio ambiente, ser humano y producción de sistemas agrícolas sostenibles, económicamente viables, ambientalmente seguros y culturalmente diversos, innovando en tecnología basada en prácticas referente a ecología (PRESANCA II y FAO, 2011).

Ahora bien, en el siglo XXI se evidencia la apertura comercial a nivel mundial, imponiendo retos al sector agrícola tradicional y temores a futuro sobre el incremento y sostenimiento de la producción de alimentos y otros productos para asegurar la alimentación adecuada para la población del planeta. En Centroamérica, el desafío se centra principalmente en la demanda de alimentos seguros y de calidad, la concentración y fortalecimiento en las cadenas agro productivas comerciales más cercanas al proveedor final, además en permanente expectativa de recepcionar conocimiento sobre la oferta de aplicación de nuevas tecnologías que generen la efectividad en la comunicación y el desarrollo del conocimiento en aras de defender el patrimonio sanitario y el compromiso con la 
preservación del medio ambiente (Gómez, Le Coq y Samper, 2014). A lo anterior se suman los diversos episodios de amenazas naturales, lo que afecta directamente al sector agrícola en el área rural y, por ende, la comercialización (Cuéllar, Davis, Luna y Díaz, 2012; Cortez-Dávila, 2016).

A pesar de las circunstancias descritas, los modelos de agricultura presentados en los diferentes Estados centroamericanos, se caracterizan por generar empleo y producción de alimentos distribuidos a la población urbana y rural, estableciendo una cadena comercial a nivel territorial e internacional, exponiendo transformaciones relevantes en el escenario económico, social y político, proyectándose a reducir los índices de pobreza, aprovechando plenamente el potencial de los suelos fértiles, motivando el fortalecimiento y la unión de las estructuras productivas e impulsando los mercados agrícolas centroamericanos. Lo anteriormente descrito, permite vislumbrar un horizonte de avance para el próximo decenio, en la proyección de indicadores de cumplimento al sector agrícola, previendo la producción de granos básicos relacionados con la seguridad alimentaria de los territorios, con la participación de pequeños productores centroamericanos, al tiempo que se elaboren y se implementen estrategias sociales relacionadas con el turismo y servicios ambientales. En este punto, se hace importante aprovechar las relaciones en términos económicos, transferencia de conocimiento que se establezcan con los tratados de libre comercio, con miras a mejorar el uso del suelo y la participación de todos los agricultores (Gómez et al., 2017).

Por otra parte, en lo concerniente al caso colombiano, se evidencia el "fomento desde las políticas públicas" hacia la agricultura campesina y la agroecología, del mismo que una dinámica de desarrollo y construcción de escenarios (biofísicos) donde el territorio juega un papel importante, dado que se configuran elementos sociales, culturales, productivos, económicos y ambientales que establecen heterogeneidades en la comunidad rural. El resultado de lo expuesto no sólo ha facilitado la movilización y puesta en marcha de experiencias a nivel productivo en diversos lugares del país, el trabajo en red, la sostenibilidad financiera, seguridad alimentaria, sino también ha expuesto la posibilidad de generar un sistema de producción bajo principios que priorizan lo solidario, la democracia, la vida, bienestar y la sustentabilidad.
Ahora bien, en Colombia la tenencia de la tierra y el agro siguen siendo un problema (a pesar de las diez reformas agrarias persiste el fenómeno), tanto para los agricultores, como para los productores agroecológicos. Es de resaltar que determinadas universidades se han venido articulando, brindando un reconocimiento a los procesos de la agroecología y sus apuestas a nivel productivo, participación, metodologías y unidades agrarias (Machado-Vargas et al., 2015).

La literatura ha demostrado que algunos de los puntos álgidos de la agroecología son: la sostenibilidad, comercialización y sustentabilidad, factores que influyen en su posicionamiento (Machado-Vargas et al., 2015) -la diferencia entre sustentabilidad y sostenibilidad radica en que el primero promueve la preservación, conservación y protección de los recursos naturales para la actual y futura sociedad, desconociendo dimensiones sociales, culturalesy políticas del individuo, mientras que la segunda reconoce y da énfasis a los aspectos desconocidos por la sustentabilidad con especial énfasis en el cuidado del ambiente-. Una alternativa para mejorar esta situación es la implementación de un diseño agroecológico con enfoque territorial colombiano que permita a las familias generar un nivel de resiliencia que facilite afrontar el escenario crítico en el aspecto socioeconómico del área agrícola. Por otro lado, es necesario la formación en capital humano, especialmente en la transmisión de saberes de campesino a campesino, la tecnificación, seguridad, salud en lo rural, fortalecimiento de dinámicas territoriales, así como también se requiere invertir en puertos, carreteras, y todos los medios comunicativos y de tecnología que permitan la accesibilidad a productores, la incursión a otros mercados reduciendo costos en la producción con intermediarios y la generación de asociaciones o redes de consumidores que opten por la producción limpia.

\section{Conclusiones}

En la historia del país, la agricultura ha jugado un papel fundamental en la dinámica económica del país. Sus avances o sus retrocesos han afectado seriamente el crecimiento del Producto Interno Bruto -en adelante PIB-. El sector agrario ha sido objeto de discusión permanente, de polémicas y de luchas internas relacionadas con el asunto de la tenencia de la tierra y modelos de producción. 
Por un lado, están los terratenientes, propietarios de grandes extensiones del territorio, quienes configuraron cierta concepción de feudalismo moderno que se consolidó en la acumulación de capital, teniendo como mano de obra al campesino siervo sin tierra, que tuvo que asumir el conflicto entre dos protagonistas: el propietario que se defiende con ejércitos privados para mantener su hegemonía sobre el territorio y la subversión armada que consideró siempre que la tierra es para quien la trabaja y produce con ella. De otro lado, ese mismo campesino tuvo que vivir de cerca el conflicto entre guerrilla y paramilitarismo; entre las fuerzas oficiales y las fuerzas por fuera de la ley, incluyendo, además de las mencionadas, las bandas criminales conformadas para defender los cultivos ilícitos de los narcotraficantes. Bajo ese manto oscuro de la historia, los aportes del sector agropecuario se fueron disminuyendo y el campo se volvió peligroso. En los últimos años ha disminuido la población campesina en Colombia, y en la última década su aporte al PIB se hizo inferior al 10\% (Piñeros, 2015).

En otra dimensión de la historia de la agricultura, se ha evidenciado una preponderancia de la agroindustria, caracterizada por la tecnificación de monocultivos propios de la región, tales como el plátano, banano; sorgo, maíz, arroz, palma africana; papa y yuca, entre otros productos. Sin embargo, ante el conflicto, esa misma producción también pagó las consecuencias con acciones puntuales que la afectaron, como el desplazamiento masivo de campesinos, razón por la cual los productores rurales tuvieron que asumir decisiones menos riesgosas con dificultades para la inversión y producción, lo que generó el abandono de su misión esencial en el campo (Arias y Ibáñez, 2012). Se impusieron entonces importantes restricciones al desarrollo económico del país (Junguito, Perfetti y Becerra, 2014) y se dieron resultados desastrosos que produjeron costos adicionales que le hicieron un daño a la producción agrícola, con destrucción de capital físico como maquinaria, vías, energía eléctrica. A eso se le suman fumigaciones que afectan al ser humano, a la diversidad, a los ecosistemas y al equilibrio de los suelos.

Ese panorama inicial de conflicto, empero, no fue óbice para que en los años 90 (no obstante, en algunas zonas y regiones como Simití en Bolivar, Cauca en el valle del Cauca, Andrés de Sotavento en Córdoba, Valle del Cauca, Sinú, Santa Rosa de
Cabal de Colombia se venían ejecutando acciones previas) se comenzara a pensar en Colombia en el desarrollo de experiencias relacionadas con la agroecología, basadas en las concepciones de los sistemas agrícolas tradicionales, con un relacionamiento entre cultivos, producción animal y manejo de recursos naturales (Wesel y Soldat, 2009), aportando enfoques alternativos sobre el tratamiento de la tierra, que se establecieron como experiencia crítica frente al modelo de producción dominante de la revolución verde. Así, se fomentaron iniciativas de productores orgánicos que conformaron organizaciones como el Instituto Mayor Campesino (IMCA), la Fundación Hogares Juveniles Campesinos, entre otras, las cuales procuraban la defensa del territorio, el establecimiento de la agricultura biológica; la lucha contra las consecuencias de hambre y pobreza para los campesinos menores generada por la agroindustria, a fin de defender la soberanía y autonomía alimentaria (Agricultura Familiar Agroecológica Campesina, 2011). Esa visión permitió el surgimiento de procesos de certificación y comercialización de productos orgánicos, con casos modelos en zonas costeras, en zonas de sabana, boscosas y planicies. Allí, en esos lugares, se fomentaron los policultivos, los huertos caseros, los sistemas de terrazas; el abonamiento orgánico, el manejo integrado de plagas, la diversificación y rotación de cultivos. Sin embargo, las certificaciones se hicieron y los procesos se desarrollaron sin tener en cuenta la diversidad de los territorios y de las regiones, porque hubo ausencia de un mapeo de contextos que calculara riesgos y potencialidades. Un evento importante para anotar está relacionado con la poca participación de la academia en el proceso, a diferencia de los países que han sido mencionados. Por el contrario, el impulso fue promovido por el sector privado que financió investigaciones sobre el modelo de producción agro, pero en el afán de sacar provecho de la industria y el comercio (Toro-Pérez, 2014), más allá de las iniciativas populares que aspiraban a una construcción de la agroecología desde las bases, en una red de escuelas campesinas (Acevedo, 2011). La red de escuelas campesinas es el resultado de un proceso liderado por organizaciones sociales y campesinas que se caracteriza por la asociatividad y la poca participación de diversos sectores (academia, estado, entre otros). 
De acuerdo con Moreno (2016), la construcción de paz en el post-acuerdo requiere afrontar el conflicto ambiental, social y armado, desde el desafío político de promover la democracia, la participación efectiva en la elaboración de una agenda agraria, la garantía de la soberanía alimentaria, la dignidad y autonomía de los pueblos históricamente marginados, la protección de la naturaleza y la adaptación a las nuevas dinámicas climáticas y ecológicas. Desde esa perspectiva, el país debe asumir el compromiso teniendo en cuenta que el desarrollo técnico productivo del sector agrario en Colombia requiere de una intervención directa del Estado, a través de un desarrollo organizacional que surja desde las comunidades, pero que debe estar articulado a nivel institucional, como resultado de la concertación política en el ámbito local, regional y nacional. De otra parte, el desarrollo comercial de la puesta de los productos en el mercado no puede darse alejado de la presencia directa de los productores rurales, quienes, además, recibirán desde la academia la instrucción necesaria para adaptar el modelo agroecológico a la vida misma del suelo colombiano, con procesos de resiliencia y de reconocimiento de los valores tradicionales del saber campesino. En este punto juega un papel importante la dimensión política de la agroecología, dado que se generan espacios de empoderamiento, liderazgo, procesos sociales de acompañamiento, fortalecimiento del tejido social e iniciativas, movimientos sociales y de campesinos que trabajan en el desarrollo de alternativas (producción y distribución agrícola) que se fundamentan en la defensa de la tierra y el territorio, conservación del medio ambiente y la soberanía alimentaria.

Ahora, el asunto es ¿cómo construir una realidad que beneficie al sector agrícola? si aún persiste un modelo de desarrollo en el país que se concentra en el sector privado, en la marginalidad de los campesinos, en la explotación de recursos del sector primario de la economía, que por supuesto es depredador.

El diseño de nuevas dinámicas de desarrollo en las zonas rurales, hacia la búsqueda de una paz estable, duradera y sostenible, implica que se piense en métodos de recuperación del campo, que se conviertan en vías amplias y alternativas en el posconflicto. Para ese fin, hay que repensar los conceptos que históricamente han estado vinculados con la práctica de la agricultura como las metodologías participativas y de diálogo inter e intracomunitario, los mercados locales, modelo de desarrollo-producción (políticas locales y regionales para apoyar las iniciativas en los territorios). Se hace necesario que lo propuesto en los acuerdos de paz sobre el tema rural, incorpore en los contextos particulares nuevas formas para trabajar el agro, en consideración con las dimensiones sociales, económicas, ecológicas, políticas y tecnológicas, hasta alcanzar una renovación sistémica. Esa perspectiva supone una comprensión transdisciplinaria de la producción agrícola y una visión holística de todo lo que involucra lo agrario. Lo planteado ha sido operacionalizado por diversas organizaciones sociales y campesinas, entre las que se mencionan algunas del Magdalena Medio: Asociación de Productores Alternativos de Simití Bolivar - ASPROAS-, Asociación Municipal de Mujeres Campesinas de Lebrija "AMMUCALE", Asociación de Trabajadores Campesinos del Carare -ATCC-.

Finalmente, "la transición de la agricultura campesina hacia la sustentabilidad depende directamente de las inversiones que se hagan para mejorar la gestión social, productiva y económica, así como en los stocks de capital humano, natural productivo y social" (Yurjevic 1998, citado en Alvarado, 1 de julio de 2004, p.2). Los Programas de Desarrollo con Enfoque Territorial representa una oportunidad en el horizonte de Colombia para lograr un desarrollo rural y sociopolítico integral de las comunidades.

\section{Referencias}

Acevedo, O. (2011). Escuelas de Agroecología en Colombia. La construcción del conocimiento agroecológico en manos campesinas. Universidad Nacional de Colombia. Disponible en https://www. researchgate.net/.../281443176_Escuelas_ de_Agroecologia_en_Colombia

Agricultura Familiar Agroecológica Campesina. (2011). Agricultura familiar agroecológica campesina en la comunidad andina: Una opción para mejorar la seguridad alimentaria y conservar la biodiversidad. Perú: Pull Creativo S.R.L.

Altieri, M. \& Toledo, V. (2010). La revolución agroecológica de América Latina: Rescatar la naturaleza, asegurar la soberanía alimentaria y empoderar al campesino. El otro Derecho, 42, 163202. Disponible en www.biblioteca.clacso.edu.ar/ Colombia/ilsa/20130711054327/5.pdf 
Alvarado, F. (julio de 1993). "Crecimiento sin costo ambiental. Experiencias de agricultura ecológica”, en SEPIA V. Disponible en http:// www.sepia.org.pe/facipub/upload/cont/877/cont/ file/20080903041027ALVARADOCrecimiento_sincosto_ ambiental__.pdf

Alvarado, F. (diciembre de 2001). El impacto de la transición hacia la agricultura ecológica en la pequeña agricultura. Alto Piura Perú.

Alvarado De la Fuente, F. y Wiener, H. (1998). Ofertas agroecológicas para pequeños agricultores: doce experiencias exitosas agricultura ecológica. Lima, Perú: Centro IDEAS.

Alvarado de la Fuente, F. (2000). Siete retos de la agroecología: desde la experiencia en elmovimiento agroecológico peruano. Revista del Postgrado en Ciencias del Desarrollo CIDES-UMSA, 8, 231-239

Alvarado de la Fuente, F. (1 de julio de 2004) Movimiento de Agricultura Ecológica en el Perú: Balance 1980-2003. Disponible en https://www.alainet. org/es/active/6419

Alvarado de la Fuente, F. (9 de octubre de 2012). Fundamentos de la Agricultura Ecológica. Centro ideas: Ideas.org.pe. Disponible en http://www.ideas.org.pe/fernando/item/238fundamentos-de-la-agricultura-ecologica

Amaya-Palacios, R. (2013). Aportes de la Agricultura Familiar a la Disponibilidad de Alimento en dos Comunidades de Río San Juan. (Tesis de grado, Universidad Nacional agraria, facultad de desarrollo rural. Managua, Nicaragua). Disponible en repositorio.una.edu.ni/3175/1/ tne50a489.pdf

Arias, M. A., \& Ibáñez, A. M. (2012). Conflicto armado en Colombia y producción agrícola: ¿aprenden los pequeños productores a vivir en medio del conflicto? (44). Documentos CEDE Disponible en https://economia.uniandes.edu.co/components/ com booklibrary/ebooks/dcede2012-44.pdf

Baumeister, E. (2010). Pequeños productores de granos básicos en América Central: Cuantificación, caracterización, nivel de ingresos, pobreza, y perfiles demográficos, socioeconómicos y ocupacionales. Honduras: Comunica.

Boza-Martínez, S. (2010). La agricultura ecológica como parte de la estrategia de desarrollo rural sostenible en Andalucía. (Tesis doctoral). Universidad Autónoma De Madrid. Madrid, España.

Camacho, A. \& Mejía, D. (2014). Consecuencias de la aspersión aérea en la salud: Evidencia desde el caso colombiano. Versión en español publicada en el libro "Costos Económicos y Sociales del
Conflicto en Colombia”. Universidad de los Andes. Bogotá. Disponible en cdn.ideaspaz.org/ media/website/document/57d74f9405f4d.pdf

Caporal, F. \& Costabeber, J. (2004). Agroecologia e extensâo rural. Contribuiçoes para a promoçâo do desenvolvimiento rural sustentável. Porto alegre, Brasilia: Mda/saf/dater-iica.

Carrazón, J., Corleto, M. y Sibrián, R. (2013). Centroamérica en Cifras. Datos de Seguridad Alimentaria Nutricional. Disponible en http:// www.fao.org/3/a-at771s.pdf.

Coordinadora de Entidades Extranjeras de Cooperación Internacional (COEECI). (2014). Agricultura Familiar en el Perú garante de la seguridad alimentaria y la agrobiodiversidad. Aportes para el debate en el marco del Año Internacional de la Agricultura Familiar. Lima-Perú: Chataro Editores, Psje. Disponible en http://www.coeeci. org.pe/wp-content/uploads/2014/06/COEECIAgricultura-Familiar-en-el-Peru-garante-de-laseguridad-alimentaria-y-la-agrobiodiversidad.pdf

Cortez-Dávila, L. (2016). Contribución de los huertos familiares a la seguridad alimentaria y nutricional de las familias que participan en el Programa Agroambiental Mesoamericano de Trifinio y área centro norte de Nicaragua. (Tesis de maestría Centro Agronómico Tropical De Investigación Y Enseñanza. Turrialba, Costa Rica). Disponible en repositorio.bibliotecaorton.catie.ac.cr/.../discover?... SEGURIDAD+ALIMENTARIA

Cuéllar, N., Davis, A., Luna, F., \& Díaz, O. (2012). Inversiones y dinámicas territoriales en Centroamérica. Implicaciones para la gobernanza y la construcción de alternativas. San Salvador: PRISMA.

Denardi, R. A. (2001). Agricultura familiar e políticas públicas: Algunos dilemas e desafíos para o desenvolvimiento rural sustentable. Agroecología e desenvolvimento rural sustentável, Porto Alegre, 2(3), 56-62.

Dürr, J. (2015). Diez mitos y realidades sobre las cadenas agroalimentarias en Guatemala, y una cuenta nacional de base agraria. Revista de estudios sociales, Guatemala. 79, 2-93.

Felipe-Morales, C. (2001). La agricultura ecológica: logros, retos y posibilidades en el Perú. Lima, Perú: Edición del autor.

Fernández, L. (2011). Ministerio de Salud. POLITICA NACIONAL DE SEGURIDAD ALIMENTARIA Y NUTRICIONAL 2011-2021. Costa Rica. Disponible en https://www.ministeriodesalud. go.cr/index.php/biblioteca-de-archivos/sobre-el- 
ministerio/politcas-y-planes-en-salud/politicasen-salud/1106-politica-nacional-de-seguridadalimentaria-y-nutricional-2011-2021/file

Gálvez, J. Buch., M., Pineda, P., Carrera-Cruz, J., Ortiz, A., Carrera, J., Andrews, K., Vargas, H., RiveraDelgado, A., Iturbide, M. \& Chavería, H. (2015) Perfil del agro y la ruralidad de Guatemala 2014: situación actual y tendencias. Guatemala: Cara Parens de la Universidad Rafael Landivar.

Gómez, I., Le Coq, J. F., \& Samper, M. (2014). Las agriculturas familiares en Centroamérica: procesos y perspectivas.

Gómez, I., Cartagena, R., Ortiz, X., \& Díaz, O. (2017). La agricultura familiar campesina en Centroamérica: una apuesta estratégica frente a los desafíos de los territorios rurales. Realidad: Revista de Ciencias Sociales y Humanidades, (137), 511-525.

Guzmán-Casado, M., González de Molina M. y Sevilla Guzmán, E. (2000). Introducción a la agroecología como desarrollo rural sostenible. Madrid, España: MundiPrensa.

Instituto de Nutrición de Centroamérica y Panamá (INCAP). (1998). La iniciativa de seguridad alimentaria nutricional en Centroamérica. Disponible en http://www.incap.int/sisvan/index. php/es/acerca-de-san/conceptos/marco-referencialde-la-san

Junguito, R., Perfetti, J. y Becerra, A. (2014) Desarrollo De La Agricultura Colombiana (48). Colombia: Fedesarrollo

Machado-Vargas, M., Nicholls, C., Márquez, S. \& Turbay, S. (2015). Caracterización de nueve agroecosistemas de café de la cuenca del río Porce, Colombia, con un enfoque agroecológico. Idesia (Arica), 33(1), 69-83. https://dx.doi. org/10.4067/S0718-34292015000100008

Mejía, M. (2014). Reflexión Agroecológica. Cali, Colombia: Edición del autor.

Moreno, L. M. (2016). La agroecología como opción política para la paz en Colombia. Ciencia Política, 11(21).

Navas, M., Orellana, N. y Domínguez, L. (2000). La experiencia organizativa de las mujeres rurales en la transición post-guerra (1992-1999). El salvador: Edición Fundación Nacional para el Desarrollo -FUNDE-.

Organización de las Naciones Unidas para la Alimentación y la Agricultura (FAO). (2007). El estado mundial de la agricultura y la alimentación. Disponible en http://www.fao.org/docrep/010/ a1200s/a1200s00.htm

Pérez, E. (2001). Hacia una nueva visión de lo rural. En Giarraca, N. (Ed.), Una nueva ruralidad en América Latina (pp. 17-30). Buenos aires, Argentina: Florencia Enghel.

Piñeros-López, L. (2015). Análisis de la iniciativa "ciudades competitivas y sostenibles" del bid, en la ciudad de barranquilla, como modelo replicable para los territorios colombianos. (Tesis de maestría). Universidad Militar Nueva Granada. Bogotá.

Programa Regional de Seguridad Alimentaria para Centroamérica (PRESANCA II) y la Organización de las Naciones Unidas para la Alimentación y la Agricultura (FAO) (2011). Centroamérica en Cifras. Datos de Seguridad Alimentaria Nutricional y Agricultura Familiar. Disponible en http://www.fao.org/ fileadmin/user upload/AGRO Noticias/docs/ CentroAm\%C3\%A9ricaEnCifras.pdf

Ranaboldo, C. \& Venegas, C. (2007). Escalonando la Agroecología. Procesos y aprendizajes de cuatro experiencias en Chile, Cuba, Honduras y Perú. Ottawa, Canada: Plaza y Valdés.

Ruiz-Palacios, J. (2017) Diseño de un sistema de buenas prácticas agrícolas como estrategia para la certificación orgánica otorgada por GRUP ECOCERT, para la empresa agricultura e inversiones AGRAIN S.A.S. (Tesis de pregrado). Universidad Autónoma de occidente. Santiago de Cali.

Romero, W. (2014). La Agricultura Familiar en Guatemala. Serie Documentos de Trabajo $N^{\circ} 148$. Grupo de Trabajo: Desarrollo con Cohesión Territorial. Programa Cohesión Territorial para el Desarrollo. Rimisp, Santiago, Chile.

Rozo, S. (2015). El efecto de la aspersión sobre los cultivos y las tasas de mortalidad infantil en Colombia. Santafé de Bogotá. Disponible en www.bdigital. unal.edu.co/view/year/2015.html

Sarandón, S. \& Sarandon, R. (1993). Agroecología: bases teóricas para el diseño y manejo de Agroecosistemas sustentables. Buenos Aires: Editorial Universidad de la Plata.

Sarandón, S. (2002). El desarrollo y uso de indicadores para evaluar la sustentabilidad de los agroecosistemas. En Sarandón, S. (Ed), Agroecología. El camino hacia una agricultura sustentable. (pp. 393-414). La Plata, Argentina: Ediciones Científicas Americanas.

Sarandón, S. (2011). La Agroecología: Su rol en el logro de una agricultura sustentable. En Sarandón, S. (2011). Curso de Agroecología y Agricultura Sustentable. La Plata, Argentina. Disponible en https://eva.udelar. edu.uy/pluginfile.php/508850/mod resource/ content/1/Sarand \%C3\%B3n\%2C\%202011. Agroecolog\%C3\%ADa\%20Cap\%202.pdf

Sarandón, J. \& Flores, C. (Ed.). (2014). La agroecología: el enfoque necesario para una agricultura. sustentable. La Plata, Argentina: Edulp. Disponible en https://libros.unlp.edu.ar/index. php/unlp/catalog/book/72

Sevilla Guzmán, E. (2006). De la sociología rural a la agroecología. Barcelona: Editorial Icaria.

Super science me (6 de noviembre de 2015). Los Efectos de la Guerra en el Medio Ambiente. Documental 
Huellas de la Guerra (mensaje en un blog). Disponible en https://superscienceme.wordpress. com/2015/11/06/guerra-medioambiente/

Toledo, V. (2012). La Agroecología en Latinoamérica: tres revoluciones, una misma transformación. Agroecología 6: 37-46. Disponible en http://revistas.um.es/agroecologia/article/ view/160651/140521

Toro-Pérez, C. (2014). Los nuevos "Business Plans" de la bioprospección, la biotecnología y la investigación científica. Revista Semillas, (5354), 7-15.

Wesel, A., y Soldat, V. (2009). A quantitative and qualitative historical analysis of the scientific discipline of agroecology. International Journal of Agricultural Sustainability, 7(1), 3-18.

Wú-Guin, S. y Alvarado de la Fuente, F. (2008). Ideas para la Agricultura Ecológica Desde la vivencia en el movimiento agroecológico peruano $\mathrm{y}$ latinoamericano. Lima, Perú: IDEAS 